\title{
Mapas de Curvas Residuales para Procesos de Destilación Reactiva Multicomponente
}

\author{
Salvador Granados, Antonio F. Díaz \\ UNAM, Departamento de Matemáticas, Facultad de Química, Av. Universidad 3000, \\ Cd. Universitaria, Delegación Coyoacán, 04510 México, D. F.-México \\ (e-mail: gransalvador@gmail.com, afdiazgarcia@yahoo.com.mx)
}

Recibido Ago. 11, 2011; Aceptado Oct. 05, 2011; Versión final recibida Dic. 02, 2011

\begin{abstract}
Resumen
El presente trabajo plantea un modelo teórico novedoso que permite visualizar la evolución de las reacciones químicas involucradas en procesos de destilación reactiva que involucran sistemas multicomponentes. La matemática proporciona herramientas que posibilitan la representación de segmentos de la realidad con fines de análisis y con elementos básicos del álgebra lineal, los compuestos químicos son combinaciones lineales de los elementos naturales y las reacciones químicas que los involucran son matrices cuya representación gráfica está en un subconjunto de un espacio vectorial de dimensión L. Como caso de estudio, se construye un diagrama reactivo que permite localizar con claridad las especies químicas involucradas y su evolución temporal en un proceso de destilación reactiva por lotes. Este instrumento permite visualizar el comportamiento del sistema mediante un diagrama triangular convencional de mapas de curvas residuales reactivas.
\end{abstract}

Palabras clave: curvas residuales, destilación reactiva multicomponente, visualización gráfica, diagramas triangulares

\section{Residue Curve Maps for Multicomponent Reactive Distillation Processes}

\begin{abstract}
The present work presents a novel theoretical model that allows visualizing the chemical reactions evolution in a specific multicomponent reactive distillation process. Math provides tools to represent real processes for analysis, and with the basic elements of linear algebra chemical species and chemical reactions could be represented by linear combinations of natural elements and homogeneous equations systems, respectively. A subset of an L-dimensional space is required for the graphical representation of these mathematical objects. A reactive diagram is drawn as a study case. This diagram allows locating the involved chemical species as well as their temporary evolution through a batch reactive distillation process. Furthermore, in this triangular diagram, the behavior of that system can be visualized.
\end{abstract}

Keywords: residue curve, reactive distillation processes, graphical visualization, triangular diagrams 


\section{INTRODUCCIÓN}

El desarrollo de métodos cortos para el análisis, simulación y diseño de procesos reactivos binarios y ternarios a través de diagramas en el plano es muy extenso y está ampliamente documentado (Seader y Henley, 2006; Taylor y Krishna, 1993). Sin embargo, cuando los procesos reactivos involucran una mayor cantidad de compuestos, el problema se complica debido a que la representación gráfica de la evolución de todas las especies involucradas y sus interrelaciones requiere de modelos teóricos (espacios vectoriales) de dimensión mayor a 2; por lo que tales procesos no son susceptibles de representación a través de gráficas en el plano.

El principio de conservación de la materia para un sistema de $\mathrm{N}$ especies químicas en equilibrio que contienen $L$ elementos naturales se puede representar por la siguiente expresión:

$$
\sum_{i=1}^{N} \gamma_{i} \xi_{i}=0 \quad \text { con } \quad \xi_{i}=\prod_{j=1}^{L}\left(e_{j}\right)_{a_{j, i}}
$$

en donde $\xi_{\mathrm{i}}$ es el nombre codificado de la especie $\mathrm{i}, \mathrm{e}_{\mathrm{j}}$ es el símbolo del elemento natural $\mathrm{j}, \mathrm{a}_{\mathrm{j}, \mathrm{i}}$ representa el número de átomos del elemento natural j en la especie i y $\gamma_{i}$ es el coeficiente estequiométrico correspondiente a la especie.

La reacción química se representa con una matriz estequiométrica que incluye los coeficientes $a_{\mathrm{j}, \mathrm{i}}$, en forma tal que los $\mathrm{N}$ compuestos involucrados en las reacciones químicas representan las columnas y los $\mathrm{L}$ elementos naturales contenidos en tales compuestos representan los renglones

\begin{tabular}{c|ccccc}
\multicolumn{1}{c}{} & $\xi_{1}$ & $\cdots$ & $\xi_{\mathrm{i}}$ & $\cdots$ & $\xi_{\mathrm{N}}$ \\
\cline { 2 - 6 } $\mathrm{e}_{1}$ & $\mathrm{a}_{1,1}$ & $\cdots$ & $\mathrm{a}_{1, \mathrm{i}}$ & $\cdots$ & $\mathrm{a}_{1, \mathrm{~N}}$ \\
$\vdots$ & $\vdots$ & & $\vdots$ & & $\vdots$ \\
$\mathrm{e}_{\mathrm{j}}$ & $\mathrm{a}_{\mathrm{j}, 1}$ & $\cdots$ & $\mathrm{a}_{\mathrm{j}, \mathrm{i}}$ & $\cdots$ & $\mathrm{a}_{\mathrm{j}, \mathrm{N}}$ \\
$\vdots$ & $\vdots$ & & $\vdots$ & & $\vdots$ \\
$\mathrm{e}_{\mathrm{L}}$ & $\mathrm{a}_{\mathrm{L}, 1}$ & $\cdots$ & $\mathrm{a}_{\mathrm{L}, \mathrm{i}}$ & $\cdots$ & $\mathrm{a}_{\mathrm{L}, \mathrm{N}}$
\end{tabular}

De acuerdo con Granados-Aguilar et al (2008), el rango de esta matriz estequiométrica equivale a la suma del número $\mathrm{M}$ de vectores (elementos) linealmente independientes y el número $\mathrm{S}$ de restricciones estequiométricas

El comportamiento de un sistema reactivo se puede visualizar en un espacio vectorial de dimensión L (Marsden y Tromba, 2004; Larson y Edwards, 2010)). Los elementos naturales presentes en los $\mathrm{N}$ compuestos de las $\mathrm{R}$ reacciones independientes constituyen una base de este espacio vectorial.

Por ejemplo, considérese la reacción de isobuteno y metanol para producir eter metilterbutílico (MTBE): $\gamma_{1} \mathrm{C}_{4} \mathrm{H}_{8}+\gamma_{2} \mathrm{CH}_{3} \mathrm{OH} \leftrightarrow \gamma_{3} \mathrm{C}_{5} \mathrm{H}_{12} \mathrm{O}$. La reacción contiene 3 elementos naturales: $\mathrm{C}, \mathrm{H}$ y $\mathrm{O}$, que son la base de un espacio de dimensión 3 y su combinación lineal genera a los compuestos involucrados.

El balance atómico del sistema reactivo dicta que $\sum_{\mathrm{i}=1}^{\text {Reactivos }} \gamma_{\mathrm{i}} \mathrm{a}_{\mathrm{ik}}=\sum_{\mathrm{i}=1}^{\text {Productos }} \gamma_{\mathrm{i}} \mathrm{a}_{\mathrm{ik}}$, en donde $\mathrm{a}_{\mathrm{ik}}$ representa el número de átomos del k-ésimo elemento natural en la i-ésima especie, así se tiene el sistema:

$$
\begin{array}{rrr}
\mathrm{C}: & -4 \gamma_{1}-\gamma_{2}+5 \gamma_{3}=0 \\
\mathrm{H}: & -8 \gamma_{1}-4 \gamma_{2}+12 \gamma_{3}=0 \\
\mathrm{O}: & -\gamma_{2}+\gamma_{3}=0
\end{array}
$$


cuya matriz estequiométrica, tiene determinante nulo, lo que indica que al menos una de las ecuaciones depende de las otras dos, por lo que esta reacción química se puede representar en un subconjunto del espacio referido. Como se puede ver, existe al menos una submatriz de rango 2; de manera que el espacio de composición de la reacción contiene dos vectores; por ejemplo

$$
\left\{\mathrm{C}_{4} \mathrm{H}_{8}, \mathrm{CH}_{3} \mathrm{OH}\right\}
$$

Partiendo de esta base, es posible visualizar el comportamiento de ciertos procesos reactivos multicomponentes mediante diagramas binarios o ternarios convencionales, vía la aplicación del concepto de elemento a reacciones químicas (Pérez Cisneros et al, 1997), basado en el modelo químico (Michelsen \& Mollerup, 2004).

Esta propuesta ha sido empleada anteriormente en los trabajos teóricos de Viveros-García et al, 2005 y Granados-Aguilar et al, 2008 sobre el diseño conceptual de columnas y el análisis termodinámico de procesos de destilación reactiva para hidrodesulfuración profunda del diesel.

\section{HIDROGENÓLISIS DEL DIBENZOTIOFENO, DBT}

La reacción de hidrogenólisis del DBT $\left(T_{c}=853.65 \mathrm{~K}, p_{c}=37.59 \mathrm{~atm}, \omega=0.484\right)$ para producir bifenilo, $\mathrm{C}_{12} \mathrm{H}_{10}\left(\mathrm{~T}_{\mathrm{c}}=789.0 \mathrm{~K}, \mathrm{p}_{\mathrm{c}}=38.0 \mathrm{~atm}, \omega=0.364\right)$ y ácido sulfhídrico, $\mathrm{H}_{2} \mathrm{~S}$ es:

$$
\mathrm{C}_{12} \mathrm{H}_{8} \mathrm{~S}+2 \mathrm{H}_{2} \rightarrow \mathrm{C}_{12} \mathrm{H}_{10}+\mathrm{H}_{2} \mathrm{~S}
$$

En ésta, se considera que la etapa controlante es la reacción superficial entre el $\mathrm{H}_{2}$ y el DBT adsorbidos en el catalizador y es influenciada por la competencia en adsorción de DBT, $\mathrm{H}_{2}$ y $\mathrm{H}_{2} \mathrm{~S}$ en los sitios activos, no así por el $\mathrm{C}_{12} \mathrm{H}_{10}$ (Girgis \& Gates, 1991, Whitehurst, et al, 1998).

$$
r=\frac{\mathrm{kK}_{\mathrm{DBT}} \mathrm{K}_{\mathrm{H}_{2}} \mathrm{C}_{\mathrm{DBT}} \mathrm{C}_{\mathrm{H}_{2}}}{\left(1+\mathrm{K}_{\mathrm{DBT}} \mathrm{C}_{\mathrm{DBT}}+\mathrm{K}_{\mathrm{H}_{2} \mathrm{~S}} \mathrm{C}_{\mathrm{H}_{2} \mathrm{~S}}\right)^{2}\left(1+\mathrm{K}_{\mathrm{H}_{2}} \mathrm{C}_{\mathrm{H}_{2}}\right)}
$$

Aquí $r$ y k son, respectivamente, la rapidez y la constante de reacción del DBT (ambas en $\mathrm{mol} / \mathrm{g}$. de catalizador $\mathrm{s}$ ); $\mathrm{C}_{\mathrm{j}}$ es la concentración en $\mathrm{mol} / \mathrm{L}$ y $\mathrm{K}_{\mathrm{j}}$ el parámetro de adsorción (en $\mathrm{L} / \mathrm{mol}$ ) del compuesto j que se adsorbe en el catalizador.

Los parámetros respectivos de reacción y adsorción, en el intervalo de temperaturas de 548 a $598^{\circ} \mathrm{K}$, se obtienen por medio de las siguientes expresiones del tipo Arrhenius:

$k_{j}=\sigma e^{\frac{E}{R T}} \quad K_{j}=\tau e^{\frac{E a}{R T}}$

en donde $R$ es la constante universal de los gases $(\mathrm{J} / \mathrm{kg}$. mol. $\mathrm{K})$. Los factores y no dependen de la temperatura y las constantes $\mathrm{E}$ y Ea son las energías de activación para reacción y adsorción, sus valores se listan son los siguientes (Girgis \& Gates, 1991).

Tabla 1: Parámetros para expresiones de rapidez de reacción y adsorción.

\begin{tabular}{|l|l|c|c|}
\hline Parámetro & Compuesto & Factor , & E, Ea \\
\hline $\mathrm{k}$ & $\mathrm{DBT}$ & $7.87 \times 10^{5}$ & $-1.26 \times 10^{8}$ \\
\hline $\mathrm{K}$ & $\mathrm{DBT}$ & $1.8 \times 10^{-1}$ & $1.9 \times 10^{7}$ \\
\hline $\mathrm{K}$ & $\mathrm{H}_{2}$ & $4.0 \times 10^{3}$ & $3.5 \times 10^{7}$ \\
\hline $\mathrm{K}$ & $\mathrm{H}_{2} \mathrm{~S}$ & $7.0 \times 10^{-1}$ & $2.2 \times 10^{7}$ \\
\hline
\end{tabular}




\section{VISUALIZACIÓN DEL ESPACIO REACTIVO}

La construcción de un espacio para este sistema reactivo puede ser complicada debido a la intervención de 4 compuestos, por lo que es necesario simplificarlo. La modificación al espacio de composición, se puede efectuar a través del empleo del concepto de elemento.

Aquí se propone seleccionar el número mínimo de descomposiciones moleculares, en átomos, fragmentos o radicales que representan la reacción (Viveros-García, et al 2005). El sistema reactivo sugiere su distribución en 3 elementos que lo describen completamente y son: $A$ : fragmento orgánico del DBT $\left(\mathrm{C}_{12} \mathrm{H}_{8}\right)$; B: átomo de azufre $(\mathrm{S})$ y C: hidrógeno molecular $\left(\mathrm{H}_{2}\right)$, así:

$$
\gamma_{1} \mathrm{AB}+\gamma_{2} \mathrm{C} \rightarrow \gamma_{3} \mathrm{AC}+\gamma_{4} \mathrm{BC}
$$

Adicionalmente, se debe considerar la presencia de un disolvente inerte, elemento D.

Para cuantificar los $M$ elementos, se usa el concepto de fracción de elemento $W_{k}$, que implica la cantidad en que se encuentra el elemento $k\left(E_{k}\right)$ y la cantidad total de elemento $E_{T}$ :

$E_{k}=\sum_{i=1}^{N} A_{k, i} x_{i} \quad E_{T}=\sum_{k=1}^{M} \sum_{i=1}^{N} A_{k, i} x_{i} \quad W_{k}=\frac{E_{k}}{E_{T}}$

en donde $A_{k, i}$ es el número de elementos "naturales" k presentes en el compuesto i.

Esto permite reducir el espacio de composición a uno cuya geometría considera los 4 elementos representados por sus fracciones molares, que corresponde con un tetraedro (Figura 1), en donde los vértices representan los elementos "puros"; las aristas son mezclas de los elementos de sus vértices; los planos, mezclas de elementos A, B y $\mathrm{C}$ y los puntos internos del espacio son mezclas de los 4 elementos. Para localizar los componentes puros, se calculan sus coordenadas a partir de los valores de la fracción de elemento del componente i puro, $x_{i}=1.0 \quad x_{j \neq i}=0.0$, así, el punto correspondiente al DBT (AB) son (0.5, 0.5, 0.0, 0.0); el bifenilo, $(A C),(0.5,0.0,0.5,0.0)$; el $\mathrm{H}_{2} \mathrm{~S}$ (BC), $(0.0,0.5,0.5,0.0)$; el azufre $(C),(0.0,0.0,1.0,0.0)$ y el disolvente $(D),(0.0,0.0,0.0,1.0)$.

Representación del espacio de composición:

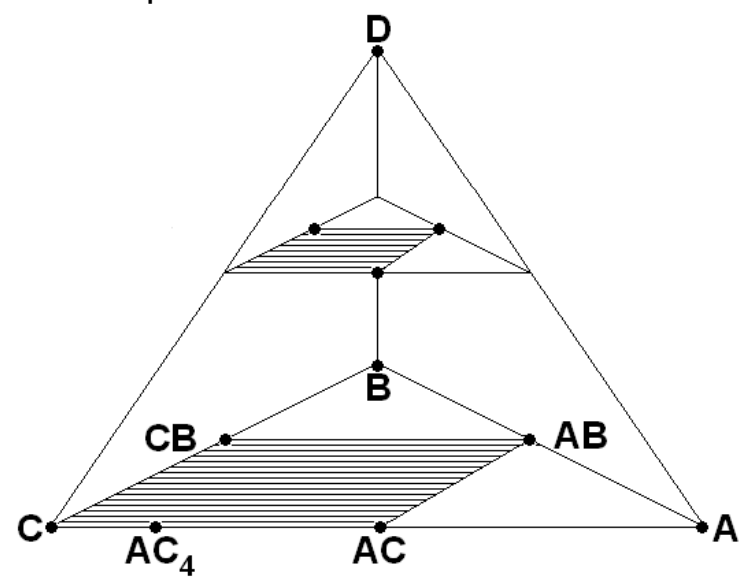

Fig. 1: Componentes puros en el espacio de composición

La región sombreada es la zona reactiva y las líneas que la delimitan se llaman fronteras reactivas; por otra parte, Bajo la consideración de las fracciones de elemento $W_{A}, W_{B}$ y $W_{C}$ "libres de disolvente", este espacio tridimensional se puede transformar en un diagrama triangular en el plano, como se muestra en la Figura 2. 


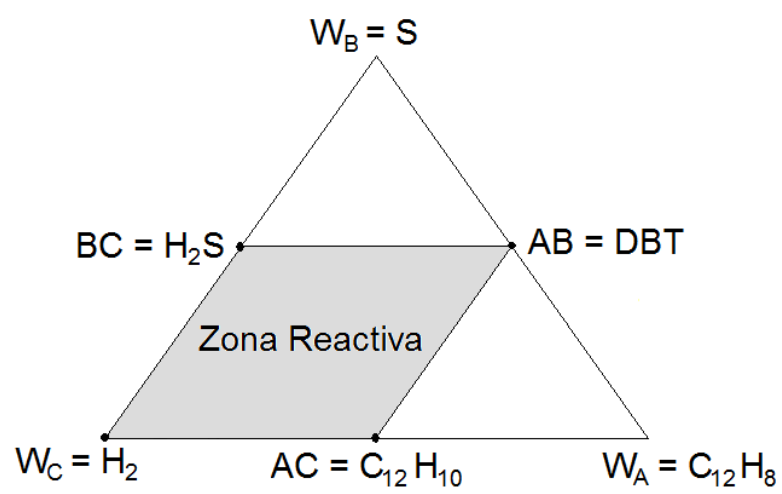

Fig. 2: Diagrama reactivo triangular normalizado libre de disolvente.

Los diagramas de fases ternarios son bastante útiles en la medida que aportan información del efecto de los parámetros del modelo sobre el comportamiento del sistema, además, permiten visualizar precisa y sencillamente el proceso de destilación reactiva.

\section{CASO DE ESTUDIO: PROCESO DE DESTILACIÓN REACTIVA POR LOTES}

La destilación reactiva es una alternativa factible para la separación de mezclas líquidas que involucran azeótropos e isómeros (Taylor \& Krishna, 2000). Su aplicación para el análisis teórico (Rivero y Herrera, 2008) de la hidrogenólisis de DBT permite observar el efecto que provoca el retiro de productos que interfieren en la conversión sobre el comportamiento de la mezcla reactiva.

Un esquema básico del proceso de destilación reactiva por lotes contiene un destilador que opera a presión $\mathrm{p}$ cargado con una cantidad $\mathrm{M}_{\text {cat }}^{0}$ de catalizador y una cantidad inicial de $\mathrm{H}_{0}$ moles de una mezcla líquida de $\mathrm{N}$ componentes, con composición $\mathrm{X}=\left(\mathrm{x}_{1}, \mathrm{x}_{2}, \ldots, \mathrm{x}_{\mathrm{N}}\right)$. El destilador se calienta mediante el suministro de una cantidad $Q$ de calor, ocurriendo así un proceso de destilación con $R$ reacciones químicas.

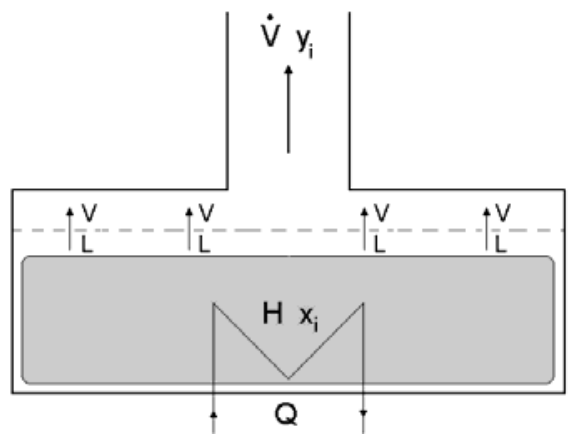

Fig. 3: Proceso de destilación reactiva por lotes

El calentamiento favorece la transferencia de masa de la fase líquida a una fase vapor, $\dot{V}=\dot{V}(t)$, cuya composición es $\mathrm{Y}=\left(\mathrm{y}_{1}, \mathrm{y}_{2}, \ldots, \mathrm{y}_{\mathrm{N}}\right)$, lo que provoca variación en la cantidad de líquido: $H=H(t)$.

Considerando que el destilador se encuentra en equilibrio líquido-vapor, su temperatura corresponde con la temperatura de burbuja del líquido, $T_{b}=T_{b}(X, p)$; así, $X$ e $Y$ representarán las composiciones de las fase líquida y vapor en el equilibrio.

El balance de materia global se establece en la forma:

$$
\frac{\mathrm{dH}(\mathrm{t})}{\mathrm{dt}}=-\dot{\mathrm{V}}(\mathrm{t})+\sum_{\mathrm{j}=1}^{\mathrm{R}} \mathrm{M}_{\mathrm{cat}}^{0} \gamma_{\mathrm{T}, \mathrm{j}} \mathrm{r}_{\mathrm{j}} ; \quad 0 \leq \mathrm{t}<\mathrm{t}_{\max }
$$


en donde $\gamma_{T, j}$ es la suma de coeficientes estequiométricos de los $N$ compuestos de la reacción $\mathrm{j}$, de rapidez $r_{j}, y t_{\max }$ es el tiempo que se requiere para la evaporación total de la mezcla.

El balance por componente resulta entonces:

$\frac{\mathrm{d}\left(\mathrm{H}(\mathrm{t}) \mathrm{x}_{\mathrm{i}}\right)}{\mathrm{dt}}=-\dot{\mathrm{V}}(\mathrm{t}) \mathrm{y}_{\mathrm{i}}+\sum_{\mathrm{j}=1}^{\mathrm{R}} \mathrm{M}_{\mathrm{cat}}^{0} \gamma_{\mathrm{i}, \mathrm{j}} \mathrm{r}_{\mathrm{j}}$

donde $\mathrm{i}=1,2, \ldots, \mathrm{N}$ y $\gamma_{\mathrm{i}, \mathrm{j}}$ es el coeficiente estequiométrico del compuesto i en la reacción $\mathrm{j}$.

Así:

$\frac{d x_{i}}{d t}=\frac{\dot{V}(t)}{H(t)}\left(x_{i}-y_{i}\right)+\sum_{j=1}^{R} \frac{M_{c a t}^{0}}{H(t)}\left(\gamma_{i, j}-x_{i} \gamma_{T, j}\right) r_{j}$

Para resolver el sistema, considere el control del tiempo de residencia mediante una estrategia de calentamiento que garantice la relación de proporcionalidad

$\frac{\mathrm{H}(\mathrm{t})}{\dot{\mathrm{V}}(\mathrm{t})}=\frac{\mathrm{H}_{0}}{\dot{\mathrm{V}}_{0}}$

e introduzca el cambio de variable

$\xi=\left(\frac{\dot{\mathrm{V}}_{0}}{\mathrm{H}_{0}}\right) \mathrm{t}$

denominado tiempo deformado, que corresponde con un tiempo característico adimensional y, por la otra, al incorporar un parámetro adimensional ( ), que describe la relación entre una rapidez de reacción característica $\left(r_{0}\right)$ y la rapidez con que las moléculas abandonan la fase líquida

$\alpha=\frac{r_{0} M_{\text {cat }}^{0}}{\dot{\mathrm{V}}_{0}}$

el modelo se transforma en:

$$
\frac{d x_{i}}{d \xi}=x_{i}-y_{i}+\alpha \frac{\dot{V}_{0}}{\dot{V}(t)} \sum_{j=1}^{R}\left(\gamma_{i, j}-x_{i} \gamma_{T, j}\right) \frac{r_{j}}{r_{0}}
$$

El equilibrio de fases se determina a partir de los coeficientes de fugacidad parcial $\left(\hat{\mathrm{f}}_{\mathrm{i}}^{v}, \hat{\mathrm{f}}_{\mathrm{i}}^{\backslash}\right)$ :

$\hat{\varphi}_{i}^{v}=\frac{\hat{f}_{i}^{v}}{y_{i} p} ; \quad \hat{\varphi}_{i}^{\prime}=\frac{\hat{f}_{i}^{\prime}}{x_{i} p}$

Las composiciones de la fase vapor en equilibrio se obtienen mediante la ecuación:

$\sum_{i=1}^{N} \frac{\hat{\varphi}_{i}^{\prime}\left(X, T_{b}\right)}{\hat{\varphi}_{i}^{v}\left(Y, T_{b}\right)} X_{i}-1=0$

La rapidez de reacción en fase líquida depende asimismo de $T_{b}$ y $X$, con lo que 


$$
\frac{d x_{i}}{d \xi}=x_{i}-y_{i}\left(X, T_{b}\right)+\alpha \frac{\dot{V}_{0}}{\dot{V}} \sum_{j=1}^{R}\left(\gamma_{i, j}-x_{i} \gamma_{T, j}\right) \frac{r_{j}\left(X, T_{b}\right)}{r_{0}}
$$

El sistema (15), para $\dot{\mathrm{V}} / \dot{\mathrm{N}}_{0}=1$ (una limitante significativa del modelo), implica la resolución de $\mathrm{N}-1$ ecuaciones diferenciales y la condición de suma de fracciones molares, por lo que se transforma en un sistema de ecuaciones algebraico-diferenciales (EAD). Su solución involucra un proceso iterativo en el cálculo de equilibrio de fases. En el programa de cómputo desarrollado en lenguaje FORTRAN para el presente trabajo, se utilizó la ecuación de estado PRSV (Stryjek y Vera, 1986).

\section{RESULTADOS}

Los mapas de curvas residuales (MCR) se usan para visualizar el comportamiento de procesos de destilación convencional, debido a que reflejan los cambios en la composición líquida durante el proceso. Este concepto se ha transferido a procesos de destilación reactiva con la correspondiente adición de modelos para velocidad de reacción, teóricos o experimentales (Venimadhavan et al, 1994; Thiel et al, 1997).

Se realizaron los cálculos para una mezcla reactiva con 500 ppm de DBT disuelto en tetralina, $\mathrm{C}_{10} \mathrm{H}_{12}$ (Granados-Aguilar et al, 2008) e hidrógeno en una relación de 10 a 1 con respecto al DBT. La presión fue de 30 atmosferas. Las curvas residuales calculadas se presentan en un diagrama ternario por elementos (Figura 4), en donde se destaca lo siguiente:

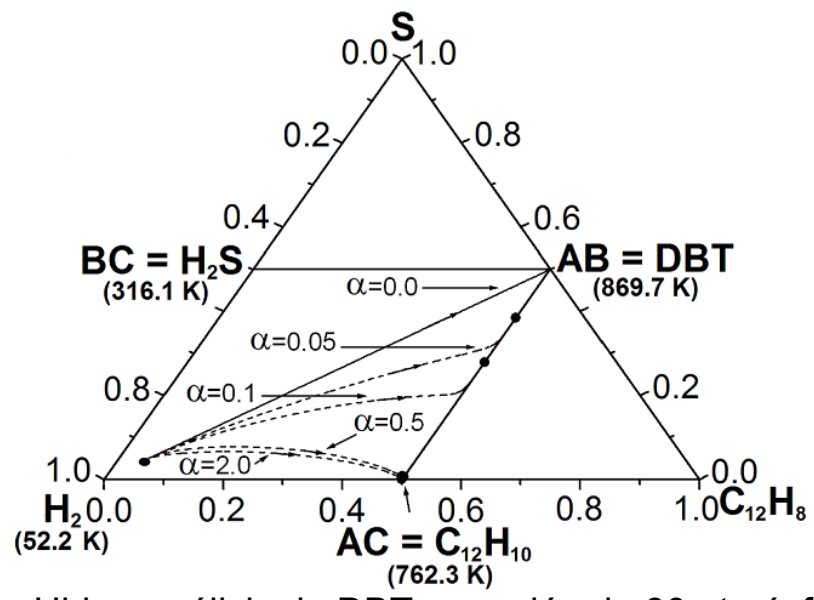

Fig. 4: Hidrogenólisis de DBT a presión de 30 atmósferas.

Las curvas describen la evolución de la fase líquida para los casos de destilación simple $(=0)$ y destilación reactiva ( $=0.05,0.1,0.5$ y 2.0); los puntos indican los límites de reacción para los que el hidrógeno se ha agotado en la mezcla reactiva. Los valores del factor , como indica la ecuación 11, están relacionadas con la masa de catalizador en el destilador; de manera que la curva $=2.0$ corresponde con la más alta carga. Es por esta razón que las curvas con $>0$ se desvían del comportamiento de la curva de destilación simple hacia el punto AC, que corresponde con el producto de hidrogenólisis, tendencia se acentúa con el incremento del factor . Por otra parte, el compuesto $A C$, bifenilo, se localiza en la zona en donde $W_{B}=0$ (ausencia de elemento $B$, azufre), lo que indica que la eliminación del $\mathrm{H}_{2} \mathrm{~S}$, considerado como un veneno del catalizador, favorece la reacción.

\section{CONCLUSIONES}

La visualización gráfica de procesos multicomponentes es complicada, por lo que la disposición de conceptos que la simplifiquen resultan muy atractivos.

El modelo propuesto permite conocer la conversión en relación con la masa de catalizador o rapidez de evaporación de la mezcla en el recipiente de destilación. 
La representación a través de elementos y su visualización en diagramas de fases reactivos ternarios permiten conocer la distribución y el rendimiento de productos de reacción.

Los diagramas reactivos de fases por elementos son esquemas prácticos que permiten determinar la factibilidad misma del proceso y comprender la repercusión de las variaciones del parámetro sobre sus variables.

\section{REFERENCIAS}

Girgis M.J., y Gates B.C., Reactivities, reaction networks, and kinetics in high pressure catalytic hydroprocessing, Ind. Eng. Chem. Res., 30 pp 2021-2058 (1991)

Granados-Aguilar, A.S., Viveros-García, T y Pérez-Cisneros E.S., Thermodinamyc analysis of a reactive distillation process for deep hydrodesulfurization of diesel: Effect of the solvent and operating conditions, Chem. Eng. J., 143 pp 210-219 (2008)

Larson R. y Edwards B. H., Cálculo 2, Mc Graw Hill/Interamericana Editores S. A. de C. V. (2010)

Marsden J. E. y Tromba A. J., Cálculo Vectorial 5a Ed. Pearson Educación S. A. (2004)

Michelsen M. L. y Mollerup J. M., Thermodynamic Models: Fundamentals and Computational Aspects, Tie-Line Publications (2004)

Pérez-Cisneros E.S., Gani R. y Michelsen M. L, Reactive separation systems-I. Computation of physical and chemical equilibrium, Chem. Eng. Sci., 52 (4), pp. 527-543 (1997).

Rivero P. y Herrera R., Análisis de Modelos Cinéticos de Polimerización vía Simulación Matemática, Inf. tecnol., [online], 2008, vol. 19, n-4, pp.25-34, ISSN 0718-0764.

Seader J. D y Henley E. J., Separation Process Principles, $2^{\text {nd }}$ Ed. John Wiley (2006)

Stryjek R. y Vera J. H., PRSV- An improved Peng-Robinson equation of state with new mixing rules for strongly nonideal mixtures, The Can. Journal of Chem. Eng. 64, pp 224-340 (1986)

Taylor R. y Krishna R, Multicomponent Mass Transfer, John Wiley (1993)

Taylor R. y Krishna, R., Modeling reactive distillation, Chem. Eng. Sci. 55, 5183 (2000).

Thiel C., Sundmacher K. y Hoffmann U., Synthesis of ETBE: Residue curve maps for the heterogeneously catalyzed reactive distillation process, Chem. Eng. J., 66 pp. 181-191 (1997)

Venimadhavan G., Buzad G., Doherty M. F. y Malone M. F., Effect of kinetics on residue curve maps for reactive distillation, AIChE, J., 40(11) pp. 1814-1824 (1994)

Viveros-Garcia, T., Ochoa-Tapia J. A., Lobo-Oehmichen R., de los Reyes-Heredia J. A. y PérezCisneros E. S., Conceptual design of a reactive distillation process for ultra-low sulfur diesel production, Chem. Eng. J. 106, pp. 119-131 (2005).

Whitehurst D. D., Isoda T. y Mochida I., Present state of the art and future challenges in the hydrodesulfurization of polyaromatic sulfur compounds, Advances in Catalysis 42, pp. 345-471 (1998) 